\title{
Analysis Of Aircraft Pitch Axis Stability Augmentation System Using Sum Of Squares Optimization
}

\author{
Kailash Krishnaswamy, George Papageorgiou, Sonja Glavaski, Antonis Papachristodoulou
}

\begin{abstract}
In this paper, we use SOS (Sum of Squares) programming approaches to analyze the stability and robustness properties of the controlled pitch axis (6 state system) of a nonlinear model of an Aircraft. The controller is a LTI Dynamic Inversion based control law designed for the short period dynamics of the Aircraft. The closed loop system is tested for its robustness to uncertainty in the location of center of gravity along the body x-axis. Results in the form of stability regions about a trim point are computed and verified using simulations.
\end{abstract}

\section{INTRODUCTION}

Design and stability analysis of an aircraft pitch axis control system has been a well studied problem, [1], [2]. Some researchers, [1], determined analytically the stability regions of a F-14 in terms of the engine thrust and pitch attitude and presented a global stability result for nonlinear modeled pitch axis of a F-14 modulated by a nonlinear dynamic inversion control law. Recently, a new computationally tractable nonlinear system analysis method was proposed, [3]. From a computation perspective, the method relaxes searching for positive semi definite stability certificate functions (eg. Lyapunov functions) to searching for sum of squares (of appropriate polynomials) certificate functions. Solving for the relaxed requirement leads to solving a SDP (Semi Definite Program) which is computationally tractable. This approach was used for Lyapunov function synthesis, [4] and estimating the stability region of SDRE (State Dependant Riccati Equation) systems, [5]. A recently developed software, SOSTOOLS(v1.01), [6] was used to convert the required sum of squares conditions to an appropriate SDP which was then solved using SeDuMi, [7]. In this paper we determine, numerically, the region of attraction of a trim point for the pitch axis of a nonlinear modeled aircraft modulated via a linear dynamic inversion based controller. The model incorporates uncertainty in the position of center of gravity along X-body axis. The stability regions are computed using SOSTOOLS.

This paper is organized as follows. Section II states the relevant results used in this paper. Due to space limitations, it is not possible to include all the details. An interested reader is referred to [8] for more details. Section III presents stability analysis results for the controlled pitch axis short period dynamics (5 states). Section IV presents stability analysis results for the controlled pitch axis longitudinal (short period + phugoid) dynamics (6 states). Conclusions and future work are presented in section V.

\section{BACKGROUND}

\section{A. Aircraft model}

In [9] the authors proposed a Robust Flight Control design benchmark problem. The problem was to design an autopilot for the final segments of an approach for a fictitious aircraft - the Robust Civil Aircraft Model

K. Krishnaswamy (kailash.krishnaswamy@honeywell.com), G. Papageorgiou and S. Glavaski are with Honeywell Labs, 3660 Technology Dr, Minneapolis, MN 55418.

A. Papachristodoulou is with the Department of Control and Dynamical Systems, California Institute of Technology, Pasadena, CA 91125.
(RCAM). A six degree of freedom nonlinear model of the RCAM including nonlinearities of actuators (thresholds) and a model of disturbances was proposed. For the purpose of this paper, we consider the nonlinear model without any disturbance inputs. The state space, we consider, is given by $[q, V, \alpha, \theta]^{T}$, which stand for the pitch rate $(\mathrm{rad} / \mathrm{s})$, Velocity $(\mathrm{m} / \mathrm{s})$, angle of attack $(\mathrm{rad})$ and pitch angle (rad), respectively. The control inputs are $\left[\delta_{e}, \delta_{T H}\right]^{T}$ which stand for the elevator deflection (rad) and throttle lever deflection (rad), respectively. More details regarding the specific model we use in this paper is given in [8].

\section{B. Stability of constrained nonlinear systems}

It is well know that a sufficient condition for ensuring that an equilibrium point of an unconstrained dynamical system is stable, is the existence of a Lyapunov function, [10]. A sufficient condition for ensuring the stability of an equilibrium point of a constrained nonlinear dynamic system was recently proposed, [4]. A simplified version of the theorem without proof is given in [8]. Loosely speaking, stability of a constrained nonlinear system was guaranteed subject to the existence of a local Lyapunov function. We present another sufficient condition for ensuring the stability of an equilibrium point in this paper.

Lemma 1: Consider a dynamic system with polynomial (possibly rational) vector field, $f(x, u)$, polynomial constraints, $a_{i}(x, u), b_{j}(x, u)$ where $x \in R^{m}, u \in R^{n}$ :

$$
\begin{aligned}
& \dot{x}=f(x, u) \\
& a_{i}(x, u) \leq 0 \\
& b_{j}(x, u)=0 .
\end{aligned}
$$

In the above equations $u$ represents all possible inputs such as control inputs, uncertain parameters, disturbances, etc. It is assumed that

○ $f(x, u)$ is nonsingular in $\mathcal{D}(\tilde{x})$, where $\mathcal{D}(\tilde{x}) \subset R^{m+n}$,

○ $f(x, u)=0$ when $x=0 \in \mathcal{D}(\tilde{x})$,

where $\mathcal{D}(\tilde{x})$ is the domain of definition of the system. $x=0$ is a stable equilibrium point of the system (1), if $\exists W(x), \psi(x), w(x, u), p_{i}(x, u), q_{j}(x, u)$ all polynomial, such that

$$
\begin{aligned}
& . W(x)-\psi(x)+\sum p_{i}(x, u) a_{i}(x, u) \\
& +\sum q_{j}(x, u) b_{j}(x, u) \text { is } \operatorname{SOS}(\Longrightarrow \geq 0), \\
& .-w(x, u) \frac{\partial W}{\partial x} f(x, u) \\
& +\sum p_{i}(x, u) a_{i}(x, u)+\sum q_{j}(x, u) b_{j}(x, u) \text { is SOS, } \\
& \text { - } w(x, u)-\epsilon \text { and } p_{i}(x, u) \text { are } \operatorname{SOS} ; \epsilon>0, \\
& . \psi(x)=x^{T} \operatorname{Diag}\left[\epsilon_{i}\right] x \text { where } \epsilon_{i}>0 .
\end{aligned}
$$

In the above equations, $\mathcal{D}(\tilde{x})$ has been incorporated as one of the inequality constraints. 


\section{Control Design}

We designed a LTI dynamic inversion based control law for the short period dynamics of the aircraft pitch axis at a particular trim configuration. The trim configuration is determined at a particular flight condition; 1) dynamic pressure, $\bar{q}=4470.29 \mathrm{~Pa}, 2$ ) level flight, $\theta=\alpha$. The controller regulates the pitch angle error, $\tilde{\theta}:=\theta-\theta^{\text {trim }}$ to the origin. By substituting the controller dynamics in the nonlinear aircraft pitch axis dynamics we get the dynamics of the closed loop aircraft pitch axis as shown below, [8].

$$
\begin{aligned}
\dot{q} & =f_{q}\left(\frac{q}{V}, \alpha, \theta, \eta_{1}, \eta_{2}, \bar{q}, X_{c g}\right) \\
\dot{V} & =f_{V}\left(\alpha, \theta, \bar{q}, X_{c g}\right) \\
\dot{\alpha} & =f_{\alpha}\left(\frac{q}{V}, \frac{1}{V}, \alpha, \theta, \eta_{1}, \eta_{2}, \bar{q}, X_{c g}\right) \\
\dot{\theta} & =q \\
\dot{\eta_{1}} & =f_{\eta_{1}}\left(q, \theta, \eta_{1}, \eta_{2}\right) \\
\dot{\eta}_{2} & =f_{\eta_{2}}(q, \theta)
\end{aligned}
$$

where $q$ is the aircraft pitch rate, $V$ is the airspeed, $\alpha$ is the angle of attack, $\theta$ is the pitch angle, $\eta_{1,2}$ are the controller states, $\bar{q}$ is the dynamic pressure and $X_{c g}$ is the cg position of the aircraft in the body $\mathrm{x}$-axis. We will determine the stability and robustness (to uncertainty in $X_{c g}$ position) of a) the short period dynamics and b) short period + phugoid dynamics of the closed loop aircraft, described in Eq. (2), in sections III and IV, respectively.

\section{Stability ANALYSIS : SHORT PERIOD DYNAMICS}

In this section, we present the results of estimation of a stability region for the controlled (stability augmented) short period dynamic model of the Aircraft. The dynamics

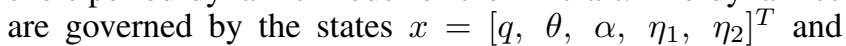
the corresponding vector fields as given in (2). The stability regions are estimated for a) the nominal closed loop system where $X_{c g}=0.23 \bar{c}$ and b) the perturbed (parameterized) closed loop system where $X_{c g} \in[0.15 \bar{c}, 0.31 \bar{c}]$.

\section{A. Stability regions of the nominal closed loop system}

The unique real equilibrium point of the short period dynamics (Eq. (2)) at a flight condition, 1) $\bar{q}=4470.29 P a$, 2) $V=90 \mathrm{~m} / \mathrm{s}$ assuming a nominal $X_{c g}=0.23 \bar{c}$, is given by:

$$
x^{*}=\left[\begin{array}{c}
q^{*} \\
\alpha^{*} \\
\theta^{*} \\
\eta_{1}^{*} \\
\eta_{2}^{*}
\end{array}\right]=\left[\begin{array}{c}
0 \\
0.01115 \mathrm{rad} \\
0.01115 \mathrm{rad} \\
0 \\
0
\end{array}\right]
$$

We transform the states, $x \rightarrow \tilde{x}=x-x^{*}$, where $x=$ $\left[q, \theta, \alpha, \eta_{1}, \eta_{2}\right]^{T}$. Using the transformed dynamics

$$
\left[\begin{array}{c}
\dot{q} \\
\dot{\tilde{\alpha}} \\
\dot{\tilde{\theta}} \\
\dot{\eta}_{1} \\
\dot{\eta}_{2}
\end{array}\right]=\left[\begin{array}{c}
f_{q}\left(q, \tilde{\alpha}, \tilde{\theta}, \eta_{1}, \eta_{2}\right) \\
f_{\tilde{\alpha}}\left(q, \tilde{\alpha}, \tilde{\theta}, \eta_{1}, \eta_{2}\right) \\
q \\
f_{\eta_{1}}\left(q, \tilde{\theta}, \eta_{1}, \eta_{2}\right) \\
f_{\eta_{2}}(q, \tilde{\theta})
\end{array}\right],
$$

we can now pose the SOS optimization problem. The formulation is as follows:

Prog 1: Given a) $\mathcal{D}(\tilde{x})=\tilde{x}^{T} \tilde{x}-\gamma^{2}$ and b) $\psi(\tilde{x})=$

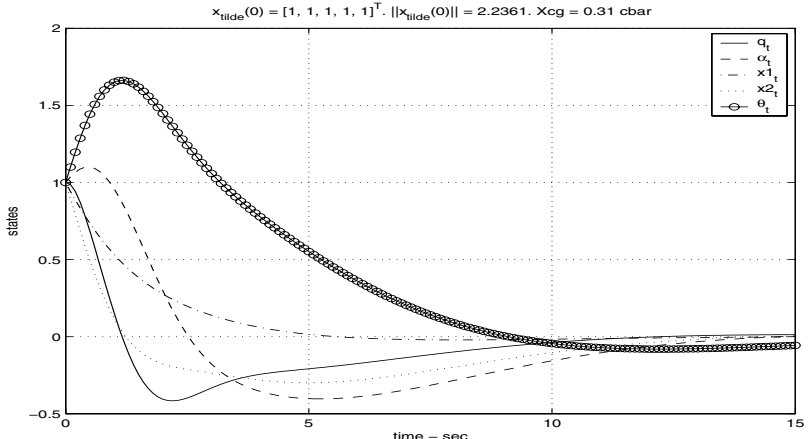

Fig. 1. $\tilde{x}$ initialized to indicate simultaneous perturbations in all states. Perturbation size $=2.236 . \gamma=2.236 / \sqrt{5}=1$

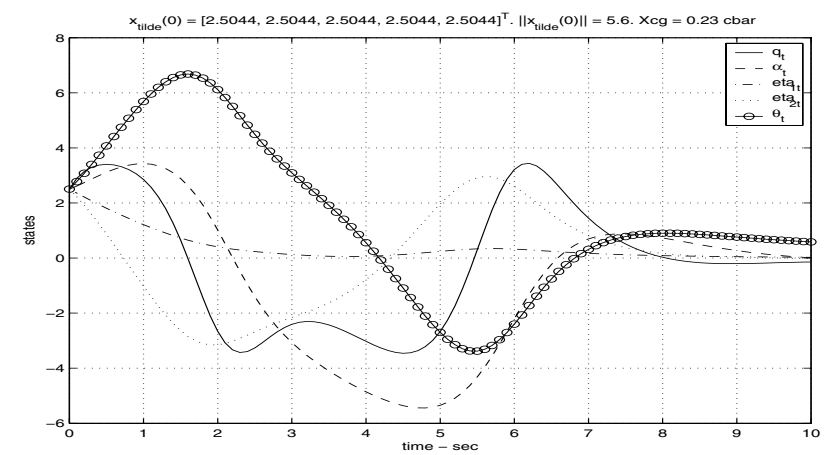

Fig. 2. $\tilde{x}$ initialized to indicate simultaneous perturbations in all states. Perturbation size $=2.5044 . \gamma=2.5044 \sqrt{5}=5.60$

$\tilde{x}^{T} \operatorname{Diag}_{i=1: 5}\left[\epsilon_{i}\right] \tilde{x}$, do there exist $W(\tilde{x}), \epsilon_{i}, p_{1}(\tilde{x}), p_{2}(\tilde{x})$ all SOS such that:

$$
\begin{aligned}
& \text { 1. } W(\tilde{x})-\psi(\tilde{x})+p_{1}(\tilde{x}) \mathcal{D}(\tilde{x}) \text { is SOS and } \\
& \text { 2. }-\frac{\partial W(\tilde{x})}{\partial \tilde{x}}\left[f_{q} f_{\tilde{\alpha}} q f_{\eta_{1}} f_{\eta_{2}}\right]^{T}+p_{2}(\tilde{x}) \mathcal{D}(\tilde{x}) \text { is SOS. }
\end{aligned}
$$

If the problem, Prog 1 , is feasible, then $\mathcal{D}(\tilde{x})$ is an estimate of the stability region of the short period dynamics about the equilibrium point (3). By solving the above feasibility problem recursively, it is possible to determine the largest $\gamma(=5.18$ in the present case $)$ such that the SOS program is feasible. Note that the stability region, $\mathcal{D}(\tilde{x})$ is the largest spherical domain of attraction of the equilibrium point (3). Via a more rigorous parametrization of $\mathcal{D}(\tilde{x})$ and a line search on $\gamma$, it may be possible to find a domain $\mathcal{D}_{1}(\tilde{x})$ which can embed the spherical $\mathcal{D}(\tilde{x})$ that we have determined. The Lyapunov function that guaranteed the local stability of the equilibrium point (3) is quadratic. A plot of the response of the controlled aircraft with initialization within the stability region $(\gamma=1<5.18)$ is shown in Fig. 1.

We present simulation results which verify the above analysis. In order to test the validity of the computed stability regions, we initialize the states of the nonlinear controlled short period dynamics Aircraft model, $\tilde{x} \in$ ext $\mathcal{D}(\tilde{x})$. The control law acts to drive the perturbed system states, $\tilde{x}$ to the origin. Four different simulations were performed. State evolution is shown in Fig. 2-5. In all four cases, the initial conditions of the states are perturbed by the largest stabilizing perturbation. Hence, any perturbation larger than that used in the simulations would destabilize the pitch axis. We can infer from the 'stable' simulation top left plot (Fig. 2) that the linear dynamic inversion 


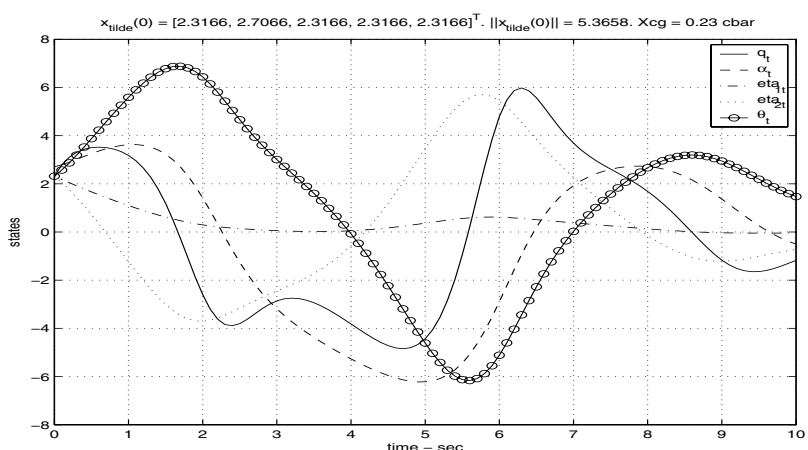

Fig. 3. $\quad \tilde{\alpha}$ initialized with largest stabilizing perturbation, $\tilde{\alpha}=2.7066$. Other states are initialized at $\frac{5.18}{\sqrt{5}}=2.3166$ (radius of the spherical $\mathcal{D}(\tilde{x})$ computed by SOSTOOLS).

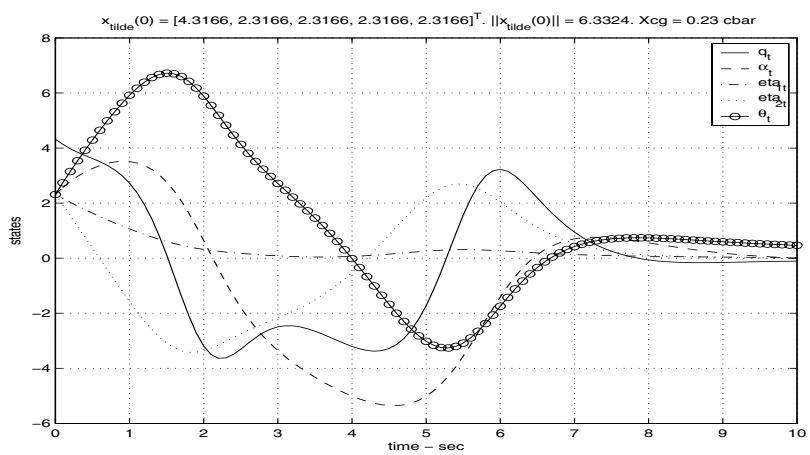

Fig. 4. $q$ initialized with largest stabilizing perturbation, $q=4.3166$. Other states are initialized at $\frac{5.18}{\sqrt{5}}=2.3166$

based controller was able to stabilize the nonlinear short period dynamics of the Aircraft even if all the states were simultaneously perturbed such that $\gamma=5.6$ which is larger than that computed using SOSTOOLS $(\gamma=5.18)$. This is because, the parametrization of $\mathcal{D}(\tilde{x}), V$ may not have been rich enough to determine the exact region. The currently computed $\mathcal{D}(\tilde{x})$ is the stability region given a quadratic lyapunov function. It may be possible to improve the estimate of the region if we considered higher degree candidates as lyapunov functions, at the cost of computation complexity. Note from both the bottom plots (Fig. 4, 5) that the control can sustain larger magnitude initializations in pitch rate $(=4.31 \mathrm{rad} / \mathrm{s}$ instead of $2.3166 \mathrm{rad} / \mathrm{s}$ as computed by SOSTOOLS) and pitch angle $(=3.81 \mathrm{rad}$ instead of $2.3166 \mathrm{rad}$ as computed by SOSTOOLS). Fig. 3 shows that the controller was not able to sustain as large perturbations in the angle of attack $(=2.71 \mathrm{rad} / \mathrm{s}$ instead of $2.31 \mathrm{rad} / \mathrm{s}$ as computed by SOSTOOLS). The fact that the closed loop system is marginally stable for larger perturbations in one state (4.31 rad/s in pitch rate) and smaller size perturbation in another state (2.71 rad in angle of attack) suggests that the region of attraction is not spherical. A richer characterization (an ellipsoid, for example) of the domain $\mathcal{D}(\tilde{x})$ could be included in the search for a higher degree lyapunov function using SOSTOOLS in order to better estimate the region of attraction. The cost would be computation complexity.

B. Stability regions of the $X_{c g}$ parameterized closed loop system

The parameterized (in terms of $X_{c g}$ ) equilibrium point of the short period dynamics at a flight condition, 1) $\bar{q}=$

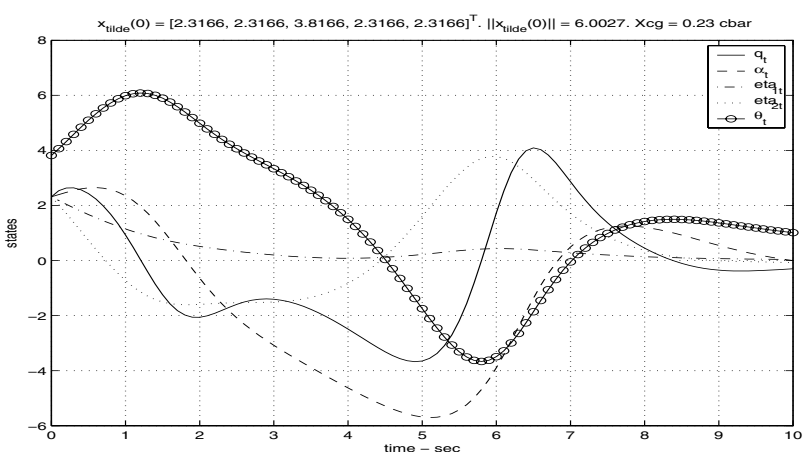

Fig. 5. $\tilde{\theta}$ initialized with largest stabilizing perturbation, $\tilde{\theta}=3.8166$. Other states are initialized at $\frac{5.18}{\sqrt{5}}=2.3166$.

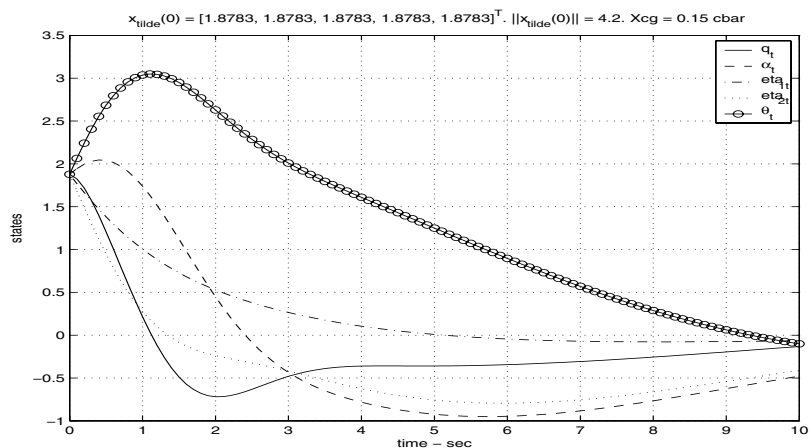

Fig. 6. Perturbation of $\tilde{x}(0)$ with $X_{c g}=0.15 \bar{c}$. Each state is perturbed by 1.8783 resulting in $\gamma=1.8783 \sqrt{5}=4.2$. This is the largest stabilizing perturbation.

$4470.29 P a, 2) V=90 \mathrm{~m} / \mathrm{s}$ is given by:

$$
x^{*}=\left[\begin{array}{c}
q^{*}=0 \\
\alpha^{*}=0.0224-0.0074 X_{c g} \mathrm{rad} \\
\theta^{*}=0.0224-0.0074 X_{c g} \mathrm{rad} \\
\eta_{1}^{*}=10^{-5}\left(-1.6+2.1 X_{c g}-0.7 X_{c g}^{2}\right) \mathrm{rad} \\
\eta_{2}^{*}=10^{-4}\left(-1.1+1.4 X_{c g}-0.5 X_{c g}^{2}\right) \mathrm{rad}
\end{array}\right] .
$$

As explained in section III-A we transform the states,

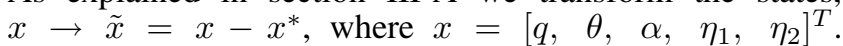
We assume here that the variation in $X_{c g}$ is significantly slower than the evolution of the short period states hence letting us assume that $\dot{X}_{c g}=0$. Following the rest of the

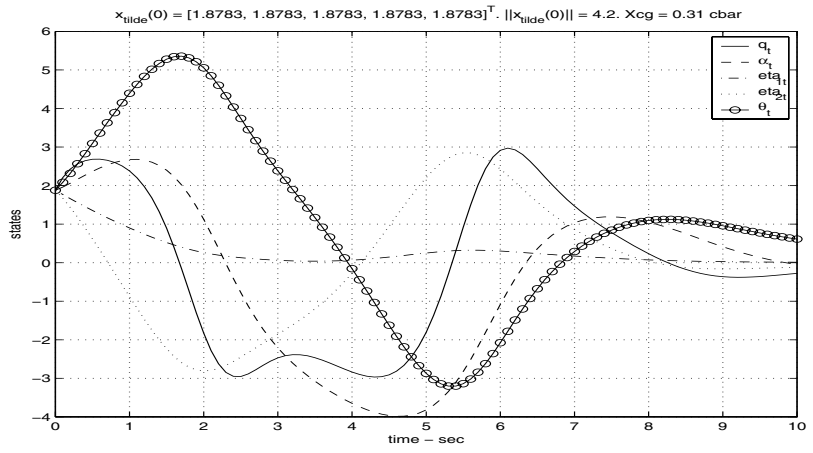

Fig. 7. Largest stabilizing perturbation of $\tilde{x}(0)$ with $X_{c g}=0.31 \bar{c}$. Each state is perturbed by 1.8783 resulting in $\gamma=1.8783 \sqrt{5}=4.2$ 


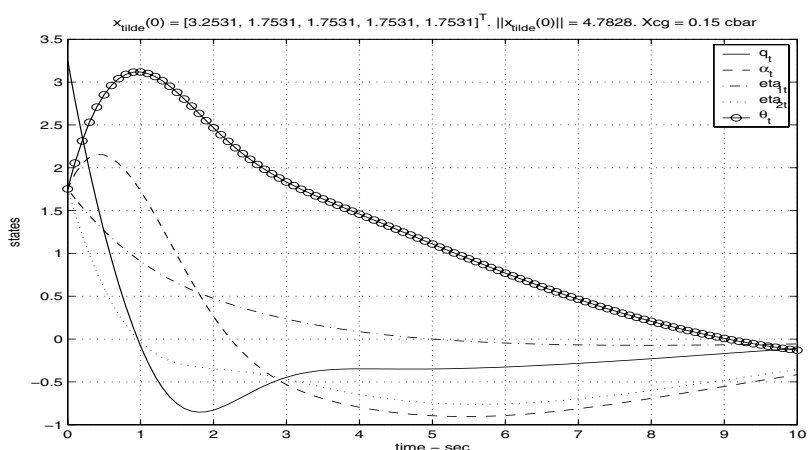

Fig. 8. Perturbation of $q(0)$ with $X_{c g}=0.31 \bar{c}$. The other states are initialized such that the perturbation size is $\frac{3.92}{\sqrt{5}}=1.7531$. This is the largest stabilizing perturbation size.

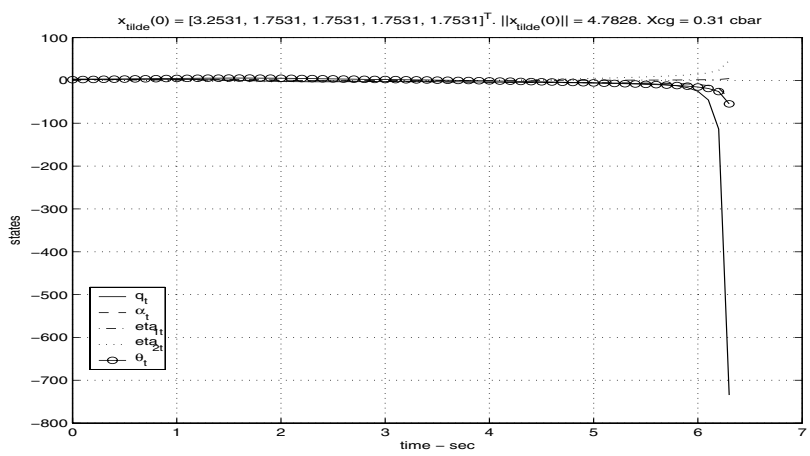

Fig. 9. Smallest destabilizing perturbation of $q(0)$ with $X_{c g}=0.31 \bar{c}$. The other states are initialized such that the perturbation size is $\frac{3.92}{\sqrt{5}}=$ 1.7531. (radius of $\mathcal{D}(\tilde{x})$ )

formulation as explained in section III-A, we can pose the SOS optimization problem. The formulation is as follows:

Prog 2: Given a) $\mathcal{D}(\tilde{x})=\tilde{x}^{T} \tilde{x}-\gamma^{2}$, b) $\psi(\tilde{x})=$ $\tilde{x}^{T} \operatorname{Diag}_{i=1: 5}\left[\epsilon_{i}\right] \tilde{x}$ and c) $C_{1}\left(X_{c g}\right)=\left(X_{c g}-0.15 \bar{c}\right)\left(X_{c g}-\right.$ $0.31 \bar{c})$ does there exist $W\left(\tilde{x}, X_{c g}\right), \epsilon_{i}, p_{j}\left(\tilde{x}, X_{c g}\right) ; j=$ $1: 4$ all SOS such that:

$$
\begin{aligned}
& \text { 1. } W\left(\tilde{x}, X_{c g}\right)-\psi(\tilde{x})+p_{1}\left(\tilde{x}, X_{c g}\right) \mathcal{D}(\tilde{x}) \\
& +p_{2}\left(\tilde{x}, X_{c g}\right) C_{1}\left(X_{c g}\right) \text { is SOS and } \\
& \text { 2. }-\frac{\partial W}{\partial \tilde{x}}\left[f_{q} f_{\tilde{\alpha}} q f_{\eta_{1}} f_{\eta_{2}}\right]^{T}+p_{3}\left(\tilde{x}, X_{c g}\right) \mathcal{D}(\tilde{x}) \\
& +p_{4}\left(\tilde{x}, X_{c g}\right) C_{1}\left(X_{c g}\right) \text { is SOS. }
\end{aligned}
$$

If the problem, Prog 2, is feasible, then $\mathcal{D}(\tilde{x})$ is a parameter independent estimate of the stability region of the short period dynamics about the equilibrium point (4). $W$ is the parameterized quadratic lyapunov function. By recursively solving Prog 2 for various values of $\gamma$, the largest spherical $\mathcal{D}(\tilde{x})$ was determined at $\gamma=3.92$. A plot of the response of the controlled Aircraft with initialization within the stability region $(\gamma=1<3.92)$ is shown in Fig. 10.

The four different scenarios which are simulated are shown in Fig. 6-9. Notice from the plots (Fig. 6, 7) that the linear dynamic inversion based controller was able to guarantee stability of the closed loop system for simultaneous perturbations in states less than $\gamma=4.2$ for extreme $X_{c g}$ conditions. It was also found (simulation results not shown) that the controller stabilized the closed loop system for a finite set of $X_{c g}$ variations within the permissible limits of $X_{c g} \in[0.15 \bar{c}, 0.31 \bar{c}]$. However, given the parametrization

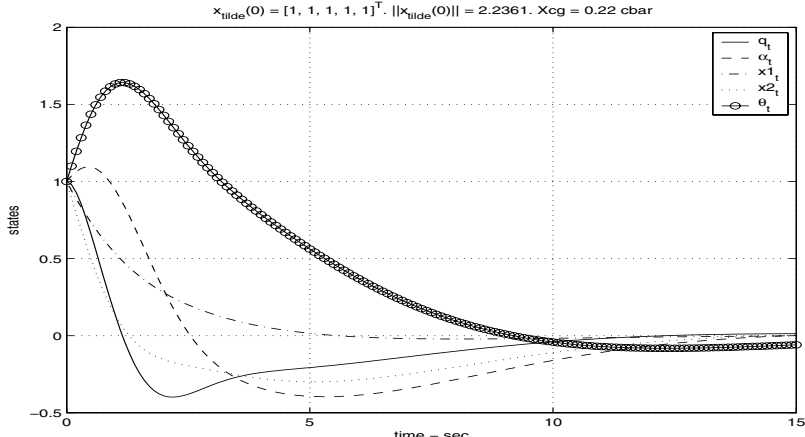

Fig. 10. Perturbation of $\tilde{x}(0)$ with $X_{c g}=0.22 \bar{c}$. Each state is perturbed by $\sqrt{5}$ resulting in $\gamma=1$.

of $\mathcal{D}(\tilde{x})$ and the Lyapunov function, since SOSTOOLS was not able to solve the posed optimization problem, it is not possible to claim that the controller will stabilize the closed loop system for perturbations that lie in $\mathcal{D}(\tilde{x})$ with $\gamma=4.2$. We can only claim that the stability region for the closed loop system is $\mathcal{D}(\tilde{x})$ with $\gamma=3.92$. The discrepancy between SOSTOOLS computed $\gamma$ and the simulation suggested $\gamma$ can be resolved by including higher degree parameterizations of both $W$ and $\mathcal{D}(\tilde{x})$ when solving the SOS optimization problem. The results of such analysis are not shown in the paper as the problem complexity was beyond what SOSTOOLS could currently handle. Note from comparing the two Fig. 8 and Fig. 9 that a $3.25 \mathrm{rad} / \mathrm{s}$ perturbation in the pitch rate, destabilizes the closed loop system if $X_{c g}=0.31 \bar{c}$ indicating that the stability region is a function of the uncertain parameter too, unlike the parametrization we assume to solve the problem. In the simulations it was assumed that $X_{c g}$ was fixed.

\section{STABILITY ANALYSIS : SHORT PERIOD + PHUGOID} DYNAMICS

In this section we present stability region estimates for the short period + phugoid dynamics modeled aircraft. Stability regions are computed for the nominal Aircraft model (no uncertainty in $X_{c g}$ position). Although we formulated the problem to determine stability regions for the short period + phugoid model Aircraft with $X_{c g}$ uncertainty, SeDuMi (the Semi Definite Programming solver called by SOSTOOLS) encountered memory issues on a P4, 2 GHz, 1 GB RAM machine running WindowsXP. Problem complexity in terms of degree of the polynomial vector fields is suspected to be the primary cause. Longitudinal dynamics of the Aircraft considered in the analysis in this section is given by (2) at $X_{c g}=0.23 \bar{c}$.

\section{A. Stability regions of the nominal closed loop system}

The equilibrium point of the dynamics (2) at a flight condition, 1) $\bar{q}=4470.29 P a$, assuming a nominal $X_{c g}=$ $0.23 \bar{c}$, is given by:

$$
x^{*}=\left[\begin{array}{c}
q^{*} \\
V^{*} \\
\alpha^{*} \\
\theta^{*} \\
\eta_{1}^{*} \\
\eta_{2}^{*}
\end{array}\right]=\left[\begin{array}{c}
0 \\
V_{t r} \mathrm{~m} / \mathrm{s} \\
0.01115 \mathrm{rad} \\
0.01115 \mathrm{rad} \\
0 \\
0
\end{array}\right] .
$$

Notice that the aircraft can theoretically attain equilibrium at any non-zero velocity as long as it is at the appropriate altitude so that the $\bar{q}$ flight condition is not violated. We will determine stability regions about various velocities along the equilibrium line $\mathrm{Eq}$ (5). Let $V_{t r}$ denote the equilibrium 


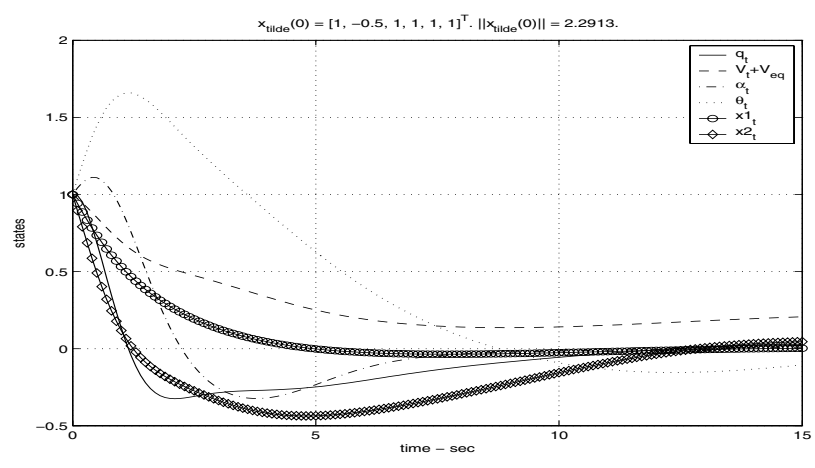

Fig. 11. $V_{t r}=150 \mathrm{~m} / \mathrm{s}, \tilde{V}=-0.5 \times 100 \mathrm{~m} / \mathrm{s}$. Therefore $V \approx 100 \mathrm{~m} / \mathrm{s}$

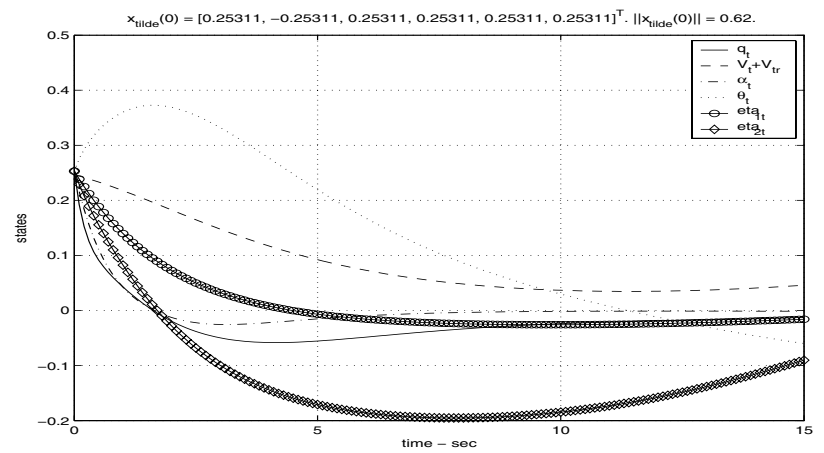

Fig. 12. $V_{t r}=50 \mathrm{~m} / \mathrm{s}, \tilde{V}=-0.25311 \times 100 \mathrm{~m} / \mathrm{s}$. Therefore $V=$ $V_{t r}+\tilde{V} \approx 25 \mathrm{~m} / \mathrm{s}$

aircraft velocity, hence $V=V_{t r}+\tilde{V}$. We transform the states, $x \rightarrow \tilde{x}=x-x^{*}$, where $x=\left[q, V, \alpha, \theta, \eta_{1}, \eta_{2}\right]^{T}$. Using the transformed dynamics

$$
\left[\begin{array}{c}
\dot{q} \\
\tilde{\tilde{V}} \\
\dot{\tilde{\alpha}} \\
\dot{\tilde{\theta}} \\
\dot{\eta_{1}} \\
\dot{\eta}_{2}
\end{array}\right]=\left[\begin{array}{c}
f_{q}\left(\frac{q}{V}, \tilde{\alpha}, \tilde{\theta}, \eta_{1}, \eta_{2}\right) \\
f_{\tilde{V}}(\tilde{\alpha}, \tilde{\theta}) \\
f_{\tilde{\alpha}}\left(\frac{q}{V}, \frac{1}{V}, \tilde{\alpha}, \tilde{\theta}, \eta_{1}, \eta_{2}\right) \\
q \\
f_{\eta_{1}}\left(q, \tilde{\theta}, \eta_{1}, \eta_{2}\right) \\
f_{\eta_{2}}(q, \tilde{\theta})
\end{array}\right],
$$

we can now pose the SOS optimization problem. The formulation is as follows:

Prog 3: Given a) the domain of definition $\mathcal{D}(\tilde{x})=$ $\tilde{x}^{T} \tilde{x}-\gamma^{2}$, b) a positive definite function $\psi(\tilde{x})=$ $\tilde{x}^{T} \operatorname{Diag}_{i=1: 5}\left[\epsilon_{i}\right] \tilde{x}$ and c) a positivity constraint on the velocity $V-\delta_{v} \geq 0$ for some $\delta_{v}>0$; does there exist $W(\tilde{x}), \epsilon_{i}, p_{1}(\tilde{x}), p_{2}(\tilde{x}), p_{3}(\tilde{x}), p_{4}(\tilde{x})$ all SOS such that:

$$
\begin{aligned}
& \text { 1. } W(\tilde{x})-\psi(\tilde{x})+p_{1}(\tilde{x}) \mathcal{D}(\tilde{x})-p_{2}(\tilde{x})\left(V-\delta_{v}\right) \text { is SOS; } \\
& \text { 2. }-V^{2} \frac{\partial W(\tilde{x})}{\partial \tilde{x}}\left[f_{q} f_{\tilde{V}} f_{\tilde{\alpha}} q f_{\eta_{1}} f_{\eta_{2}}\right]^{T}+p_{3}(\tilde{x}) \mathcal{D}(\tilde{x}) \\
& -p_{4}(\tilde{x})\left(V-\delta_{v}\right) \text { is SOS. }
\end{aligned}
$$

Note that $W(\tilde{x})$ is the Lyapunov function. In order for Eq. (7) to be a sufficient condition for stability of an equilibrium point, it is necessary that $V>0$. Hence the condition $V-\delta_{v}=$ SOS is adjoined to the above two inequalities, (7). If the problem, Prog 3 , is feasible, then $\mathcal{D}(\tilde{x})$ is an estimate of the stability region of the longitudinal dynamics about the equilibrium point (5). By solving the above feasibility problem recursively it is possible to determine, approximately,

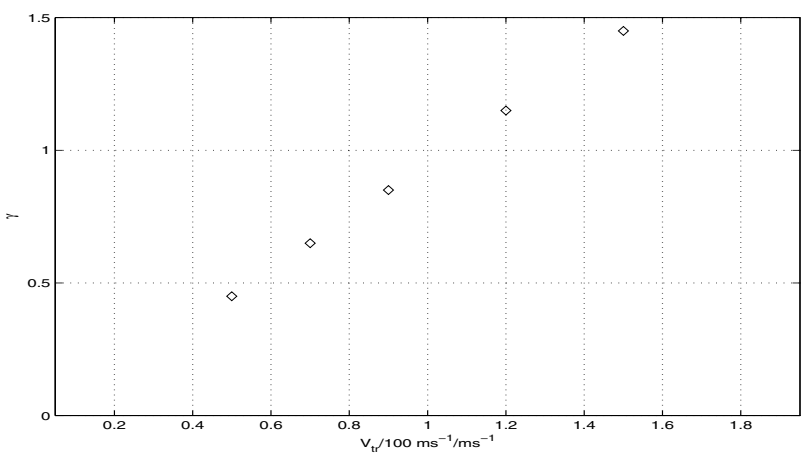

Fig. 13. $V_{t r}-\gamma$ dependence.

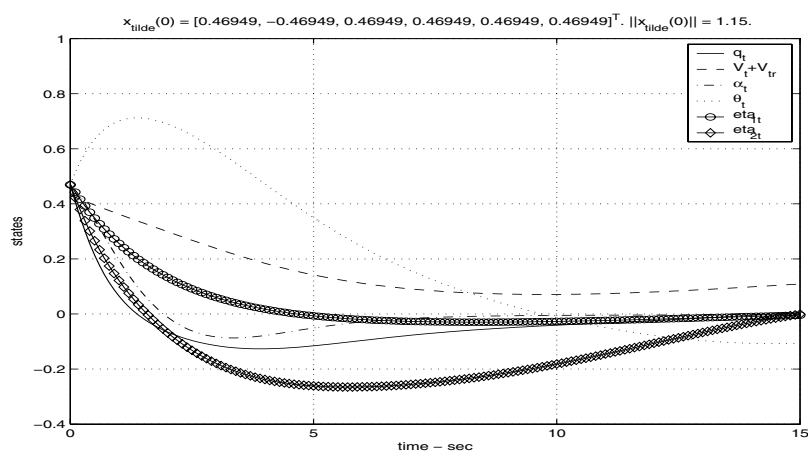

Fig. 14. $V_{t r}=90 \mathrm{~m} / \mathrm{s}, \tilde{V}=-0.46949 \times 100 \mathrm{~m} / \mathrm{s}$. Therefore $V \approx 53$ $\mathrm{m} / \mathrm{s}$

the largest $\gamma$, hence the largest stability region subject to the a) Lyapunov function and b) Domain parametrization. The system (6) displayed different sized stability domains based on the magnitude of the trim Aircraft velocity $V_{t r}$. Larger trim velocities resulted in larger estimated stability regions. A summary of the results is shown in Fig. 13. A plot of the response of the controlled Aircraft with initialization within the stability region $\left(\gamma=1, V_{t r}=150 \mathrm{~m} / \mathrm{s}\right)$ is shown in Fig. 10.

The Lyapunov function, $W(\tilde{x})$ that guaranteed the local stability of each of the equilibrium point (5) is quadratic. At an Aircraft trim velocity of $V_{t r}=90 \mathrm{~m} / \mathrm{s}$, notice that the estimated stability region had a radius of only $\gamma=0.85$. Where as, the estimated stability region at the same trim velocity assuming only short period dynamics (section IIIA) had a radius of $\gamma=5.18$. We are tempted to conclude

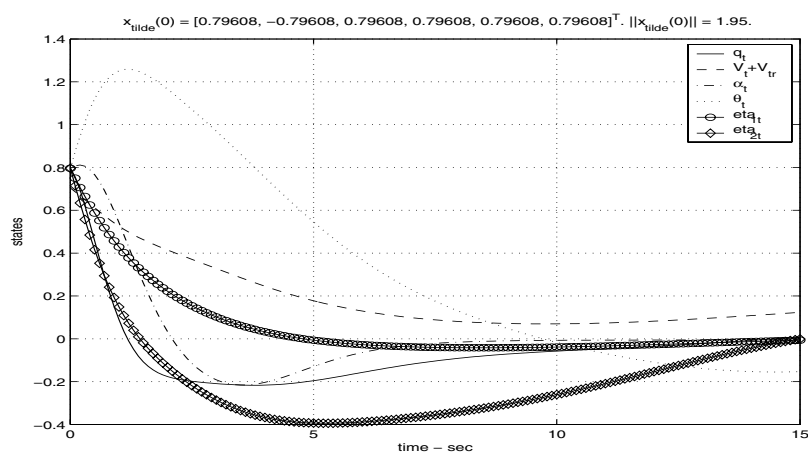

Fig. $15 . \quad V_{t r}=160 \mathrm{~m} / \mathrm{s}, \tilde{V}=-0.79608 \times 100 \mathrm{~m} / \mathrm{s}$. Therefore $V \approx 80$ $\mathrm{m} / \mathrm{s}$ 
at this point that analyzing stability of the Aircraft pitch axis considering just the short period dynamics may lead to false results such as overestimation of $\gamma$. However, let us examine the simulation results of the Aircraft dynamics (6) first. Simulations are performed by initializing the Aircraft transformed states $\tilde{x}$ such that $\tilde{x} \in$ exterior $\mathcal{D}(\tilde{x})$. The control law acts to drive the perturbed system states, $\tilde{x}$ to the origin, $\tilde{x}=0$. Plots of the responses of the states are shown in Fig. 14-15. A symbol $(\cdot)_{t}$ in the legend stands for $(\tilde{\cdot})$. Notice that in all the three simulation cases, the perturbation (initialization of $\tilde{x}$ ) size is large enough to drive the Aircraft normalized velocity, $\left(\frac{V m / s}{100 \mathrm{~m} / \mathrm{s}}\right.$, represented as $V_{t}+V_{e q}$ in the figures) close to 0 . In reality, however, pitch axis perturbations are typically never so large as to have such a significant effect on the aircraft velocity. Even if such perturbations do occur, the pilot typically uses both the elevator and throttle controls to ensure that the aircraft velocity does not decrease to stall speed (significantly higher than $0 \mathrm{~m} / \mathrm{s}$ ). Use of the throttle to ensure that the Aircraft's velocity remains close to the set trim value without significantly affecting the Aircraft attitude is possible due to the difference in time scales of the short period dynamics and the phugoid dynamics. In the simulations shown above, the throttle is trimmed to level the Aircraft and held constant during the simulation thus leading to poor performance of the stability augmentation system when perturbed simultaneously in all states.

\section{CONCLUSION AND FUtURE WORK}

In this paper, we explored the use of Sum-Of-Squares programming approaches to analyze the stability properties of a nonlinear system. Specifically, we determined quadratic stability regions (spherical) for the controlled pitch axis of a nonlinear model of an Aircraft. Stability regions were determined for a) nominal, controlled short period dynamic model, (largest $\gamma$ ) b) $X_{c g}$ uncertain, controlled short period dynamic model and c) nominal, controlled short period + phugoid dynamic model (smallest $\gamma$ ). In all the three cases, it was shown that there existed initial conditions which did not lie within the stability regions which were still stabilized by the controller. Such a result is attributed to the fact that the SOS program we solve, verifies only a sufficient condition for stability. We showed that the closed loop system's ability to remain stable to perturbations depended on the direction of the perturbation in the state space. This indicates that a spherical stability region parametrization is not the right choice to determine more accurate estimates of the region of attraction for the Aircraft pitch axis control problem. It was also found that the size of a stability region was also a function of the trim velocity of the Aircraft. Hence, an Aircraft flying at higher speed could sustain larger perturbations in the pitch axis states than an Aircraft flying at lower speeds.

The analysis presented in this paper does not assume actuator limits. We believe, that including tailplane actuator saturation limits is not only a more realistic problem but can also shrink the stability regions significantly. However, it may be challenging for SOSTOOLS (v1.01) to solve a problem with 1 or 2 additional states / higher degree vector fields. Figure 16 plots the largest dimension of the Positive Semi Definite cone (Z-axis) which contains the candidate Positive Semi Definite matrix certifying the positive definiteness of a polynomial of degree $m$, with $p$ variables. Notice the exponential growth in the worst case dimension of the cone. The time-taken to solve the programs, Prog 1,2,3 in this paper are tabulated in Tab. I. In the table, $t_{1}$ is approximately the time it takes SOSTOOLS(v1.01) to formulate the problem, $t_{2}$ is the time is takes SeDuMi to solve the formulated SDP, $n$ is the

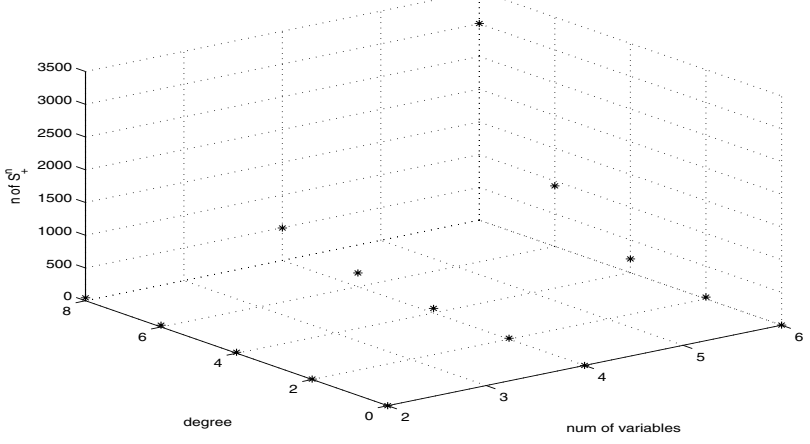

Fig. 16. Worst case dimension of the PSD cone

\begin{tabular}{|c|c|c|c|c|}
\hline poly & $\mathrm{n}($ Prog 1) & $\mathrm{n}($ Prog 2) & $\mathrm{n}($ Prog 3) & $\mathrm{n}$ (Prog 4) \\
\hline$V$ & 15 & 30 & 21 & 42 \\
$\psi$ & $5 \mathrm{i}$ & $5 \mathrm{i}$ & $6 \mathrm{i}$ & $6 \mathrm{i}$ \\
$p_{1}$ & 6 & 7 & 7 & 8 \\
$p_{3}$ & - & 7 & 28 & 8 \\
$p_{2}$ & 21 & 28 & 7 & 8 \\
$p_{4}$ & - & 28 & 7 & 8 \\
$C_{1}$ & 21 & 28 & 28 & 36 \\
$C_{2}$ & 56 & 84 & 84 & 120 \\
\hline$t_{1}(\mathrm{~s})$ & 6.64 & 28.59 & 19.71 & OOM \\
$t_{2}(\mathrm{~s})$ & 13.43 & 71.96 & 98.21 & OOM \\
\hline
\end{tabular}

TABLE I

COMPUTATION TIMES

dimension of the PSD cone and OOM is Out Of Memory. The problems were solved on a Pentium 4, 1GB RAM, $2 \mathrm{GHz}$ machine. As can be seen from Tab. I, it was not challenging to pose a 'large' unsolvable problem. In our experience, it was possible to reduce computation time and problem complexity (dimension of the psd cone) for certain problems, by choosing the right 'basis' polynomials which constitute $V(\tilde{x}), p_{i}(\tilde{x})$. However, such a method would need the control engineer to exploit the explicit structure of the polynomial vector fields. In this paper, the focus was on using SOS programming to determine approximately the stability regions of a well-studied nonlinear modeled controlled aircraft pitch axis problem. Accurate estimates of the stability regions and practical interpretation of the size of destabilizing perturbations as output by SOSTOOLS will be significant complements to the present work.

\section{REFERENCES}

[1] B. Morton, D. Enns, and B.-Y. Zhang, "Stability of dynamic inversion control laws applied to nonlinear aircraft pitch-axis models," International Journal of Control, vol. 63, no. 1, pp. 1-25, 1996.

[2] D. Enns, D. Bugajski, R. Hendrick, and G. Stein, "Dynamic inversion: an evolving methodology for fight control design," International Journal of Control, vol. 59, no. 1, pp. 71-91, 1994.

[3] P. Parrilo, "Structured semidefi nite programs and semialgebraic geometry methods in robustness and optimization," PhD Thesis, California Institute of Technology, 2002.

[4] A. Papachristodoulou and S. Prajna, "On lyapunov function synthesis using sum of squares decomposition," Proceedings of the IEEE CDC, 2002 .

[5] P. Seiler, "Stability region estimates for sdre controlled systems using sum of squares optimization," Proceedings of the American Control Conference, 2003.

[6] S. Prajna, A. Papachristodoulou, and P. Parrilo, "Introducing sostools: A general purpose sum of squares programming solver," Proceedings of the IEEE CDC, 2002

[7] J. F. Strum, "Using sedumi 1.02, a matlab toolbox for optimization over symmetric cones," Optimization Methods and Software, 1999.

[8] K. Krishnaswamy, G. Papageorgiou, S. Glavaski, and A. Papachristodoulou, "Analysis of aircraft pitch axis stability augmentation system using sum of squares optimization," Honeywell Labs Technical Report AES-R04-003, August 2004.

[9] J.-F. Magni, S. Bennani, and J. Terlouw, "Robust fight control: A design challenge," Springer-Verlag, 1997.

[10] H. K. Khalil, "Nonlinear systems," Prentice Hall, 2nd Edition, 1996. 